\title{
Is there a safe place for posterior malleolar screw fixation? An anatomic study on dry bones
}

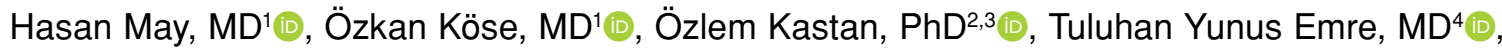 \\ Muzaffer Sindel, $\mathrm{PhD}^{2}$, Mehmet Orçun Akkurt, $\mathrm{MD}^{5}{ }^{\circledR}$ \\ ${ }^{1}$ Department of Orthopedics and Traumatology, Antalya Training and Research Hospital, Antalya, Turkey \\ ${ }^{2}$ Department of Anatomy, Akdeniz University, Medical Faculty, Antalya, Turkey \\ ${ }^{3}$ Vocational School of Health Services, Akdeniz University, Antalya, Turkey \\ ${ }^{4}$ Department of Orthopedics and Traumatology, Biruni University, Medical Faculty, Istanbul, Turkey \\ ${ }^{5}$ Department of Orthopedics and Traumatology, Ankara City Hospital, Ankara, Turkey
}

Ankle fractures with posterior malleolar (PM) involvement are not uncommon and have been reported up to $44 \% .^{[1-4]}$ It is generally accepted that a PM fracture involving the one-quarter to one-third of the distal tibial articular surface and displacement of more than $2 \mathrm{~mm}$ necessitates surgical fixation. ${ }^{[5]}$ However, recent clinical and experimental studies have shown that the fixation of the PM fracture regardless of its size provides significant stability to the ankle. ${ }^{[6-8]}$ Thus, routine fixation of all PM fractures gained acceptance among surgeons. ${ }^{[9]}$

Various techniques can be used for PM fixation, such as indirect reduction and screw fixation from the anterior approach, trans-fibular reduction and screw fixation, direct reduction and fixation from

Received: February 14, 2020

Accepted: April 23, 2020

Published online: September 11, 2020

Correspondence: Özkan Köse, MD, FEBOT. Antalya Eğitim ve Araştırma Hastanesi Ortopedi ve Travmatoloji Kliniği, 07100 Muratpaşa, Antalya, Türkiye.

E-mail: drozkankose@hotmail.com

Doi: 10.5606/ehc. 2020.73886

Citation: May H, Köse Ö, Kastan Ö, Emre TY, Sindel M, Akkurt MO. Is there a safe place for posterior malleolar screw fixation? An anatomic study on dry bones. Jt Dis Relat Surg 2020;31(3):476-479.

(02020 All right reserved by the Turkish Joint Diseases Foundation

This is an open access article under the terms of the Creative Commons Attribution-NonCommercial License, which permits use, distribution and reproduction in any medium, provided the original work is properly cited and is not used for commercial purposes (http://creativecommons.org/licenses/by-nc/4.0/).

\section{ABSTRACT}

Objectives: This study aims to determine the secure location and angle of the most distal screw during posterior malleolar (PM) fracture fixation.

Materials and methods: This prospective study was performed on September 15, 2019. Distal tibial concave articular depth and PM inclination angle were measured on 100 (50 male and 50 female) dry tibia bones using a digital caliper. A comparative analysis between male and female tibias was performed.

Results: Anteroposterior width of male tibia $(38.3 \pm 3.1 \mathrm{~mm})$ was wider than female tibia $(35.3 \pm 3.2 \mathrm{~mm})(\mathrm{p}:<0.001)$. Mediolateral length of the male tibia $(44.8 \pm 3.5 \mathrm{~mm})$ was longer than female tibia $(42.7 \pm 3.4 \mathrm{~mm})(\mathrm{p}: 0.003)$. Male tibial joint $(5.4 \pm 0.7 \mathrm{~mm})$ was deeper than that of female $(4.7 \pm 0.3 \mathrm{~mm})(\mathrm{p}:<0.001)$. The PM inclination angle was higher in male tibia $\left(18.0 \pm 1.5^{\circ}\right)$ than female tibia $\left(15.4 \pm 1.3^{\circ}\right)(\mathrm{p}:<0.001)$

Conclusion: If a screw placement parallel to the joint surface is desired, the screw insertion should be located $6 \mathrm{~mm}$ and $5 \mathrm{~mm}$ superior to the distal edge of the PM rim in males and females, respectively. If a more distal screw is required, the screw should be inserted in at least $18^{\circ}$ and $15^{\circ}$ anterosuperior direction for males and females, respectively.

Keywords: Anatomy, fracture, posterior malleolus, screw fixation, tibia.

a posterolateral approach, and direct reduction and fixation from a posteromedial approach. ${ }^{[5]}$ Each of these techniques has some advantages and disadvantages over the other. In anteroposterior (AP) fixation, the reduction is achieved without visual inspection, and the lag screws are placed from the anterior cortex under fluoroscopic control towards posterior cortex. In this technique, small fragments may not be adequately fixed. However, in posterior approach, the reduction can be visually 
monitored, and anatomic reduction can be achieved. Furthermore, it has been shown that posterior plate and screw fixation is biomechanically superior to anterior screw fixation. ${ }^{[10,11]}$ Therefore, posterior fixation technique is advocated in PM fracture fixation. ${ }^{[12]}$

The distal tibia has a concave articular surface with a slight posterior slope. The PM rim is more inferior to the anterior tibial rim. Because of this anatomic structure, there is a risk of joint penetration of screws in posterior fixations. On the other hand, there is no practical guideline to prevent screw penetration to the articular cartilage during the posterior fixation of PM fractures. In other words, to our knowledge, no anatomic study has been conducted to ensure safe screwing for this region up to date. Therefore, in this study, we aimed to determine the secure location and angle of the most distal screw during PM fracture fixation.

\section{MATERIALS AND METHODS}

This prospective study was conducted in Akdeniz University, Medical Faculty, Department of Clinical Anatomy on September 15, 2019. All dry tibia bones stored in the institution's archive were screened. Any bone that lost its distal joint integrity, or had deformity due to any reason (such as a fracture or congenital lesion) were excluded. A total of 100 (50 male and 50 female) dry tibia bones were selected from the archive and used for measurements. The study protocol was approved by the Akdeniz University,

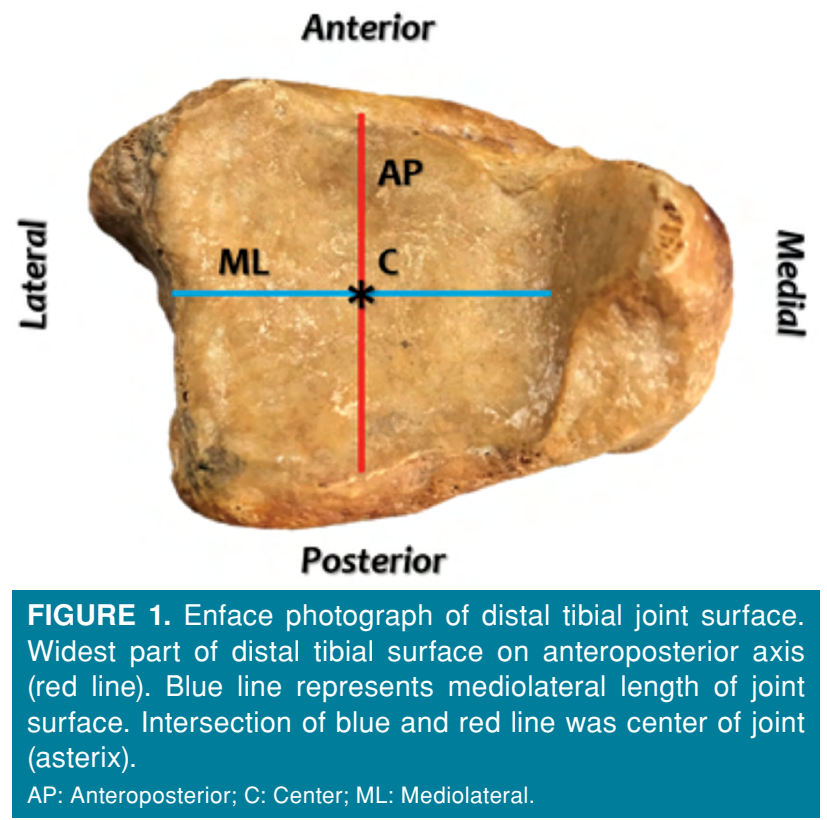

Medical Faculty Ethics Committee (Approval date and issue: 28 August 2019/792).

All measurements were performed using a digital caliper (Mitutoyo Corp., Tokyo, Japan) with $0.01 \mathrm{~mm}$ accuracy. First, the widest part of the distal joint surface of the tibia was found on the AP axis. A second line was drawn through the middle of first line and at an angle of $90^{\circ}$ on the coronal plane. The center of the concave distal tibial joint was determined (Figure 1). The depth of the joint was measured by drawing a line from the intersection of the two axes, center point, to the joint surface. An imaginary line was drawn from the endpoint of the posterior tibial rim to the deepest point of the joint to form a triangle. The angle of this triangle was measured by the AutoCAD ${ }^{\circledR}$ program (Autodesk Inc., Mill Valley, California, USA) and accepted as the angle of inclination of the posterior tibial tubercle (Figure 2). All measurements were performed twice by the same investigator (senior author) and the mean of the measurements was used for the final analysis.

\section{Statistical analysis}

Statistical analysis was performed using SPSS Statistics Base v.23 for Windows. Continuous variables were presented as mean \pm standard deviation, median, and range. The KolmogorovSmirnov test was used to determine whether the data were distributed normally. Comparative analysis of two independent groups (female and male tibias) was performed using the Student's t-test. A value of $\mathrm{p}<0.05$ was accepted as statistically significant.

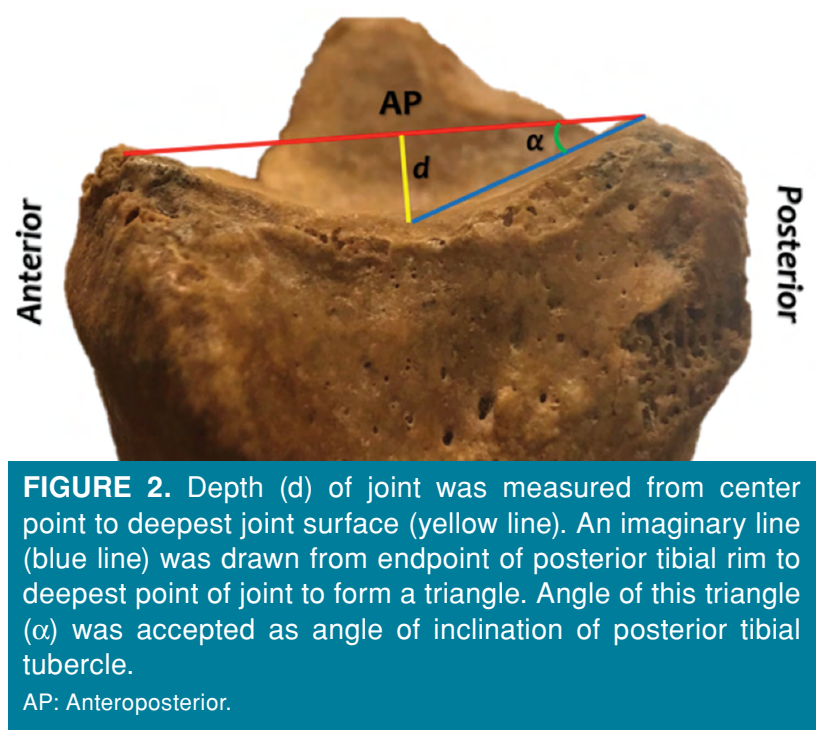




\begin{tabular}{|c|c|c|c|c|}
\hline & $\begin{array}{r}\text { TABL } \\
\text { Summary of all }\end{array}$ & Irements & & \\
\hline & All group $(n=100)$ & Male $(n=50)$ & Female $(n=50)$ & \\
\hline & Mean $\pm S D$ & Mean $\pm S D$ & Mean $\pm S D$ & $p$ \\
\hline Anteroposterior length (mm) & $36.8 \pm 3.4$ & $38.3 \pm 3.1$ & $35.3 \pm 3.2$ & $<0.001$ \\
\hline Mediolateral length (mm) & $43.7 \pm 3.6$ & $44.8 \pm 3.5$ & $42.7 \pm 3.4$ & 0.003 \\
\hline Joint depth (mm) & $5.4 \pm 0.7$ & $6.1 \pm 0.3$ & $4.7 \pm 0.3$ & $<0.001$ \\
\hline PM Inclination angle, (degrees) & $16.7 \pm 1.9$ & $18.0 \pm 1.5$ & $15.4 \pm 1.3$ & $<0.001$ \\
\hline
\end{tabular}

\section{RESULTS}

Anteroposterior width of the male tibia $(38.3 \pm 3.1 \mathrm{~mm})$ was wider than female tibia $(35.3 \pm 3.2 \mathrm{~mm})(\mathrm{p}:<0.001)$. Mediolateral length of the male tibia $(44.8 \pm 3.5 \mathrm{~mm})$ was longer than female tibia $(42.7 \pm 3.4 \mathrm{~mm})$ (p:0.003). Male tibial joint $(6.1 \pm 0.3 \mathrm{~mm})$ was deeper than female tibial joint $(4.7 \pm 0.3 \mathrm{~mm})(\mathrm{p}:<0.001)$. The PM inclination angle was higher in male $\left(18.0 \pm 1.5^{\circ}\right)$ anterosuperior (AS) direction than female $\left(15.4 \pm 1.3^{\circ}\right)$ AS direction $(\mathrm{p}:<0.001)$. The summary of all measured data is presented in Table I. Post-hoc power analysis revealed $86.1 \%$ power for the presented data.

\section{DISCUSSION}

In the current literature, many studies advocate anatomical reduction and fixation of PM fractures. ${ }^{[5-9]}$ However, very few sources provide information about how the surgical technique should be performed, and, to our knowledge, none of them provides details
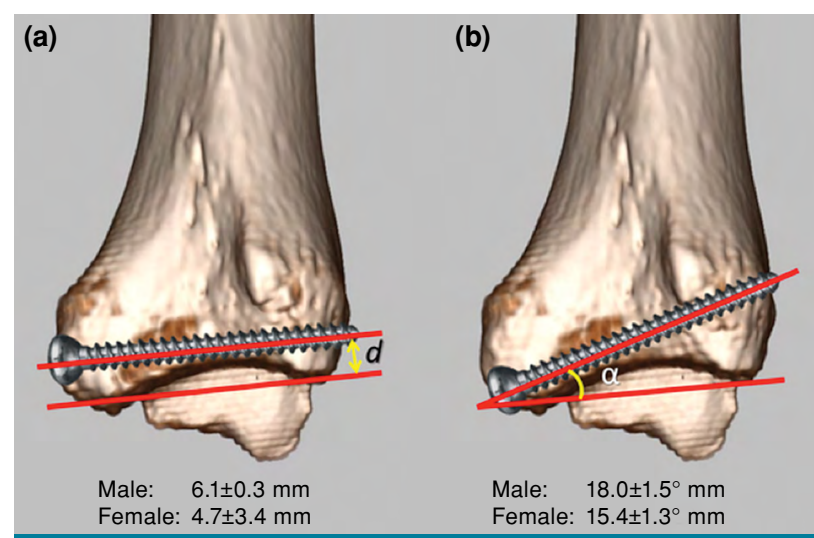

FIGURE 3. (a) Parallel screw placement to joint surface. Screw insertion point should be located $6 \mathrm{~mm}$ and $5 \mathrm{~mm}$ superior to the distal edge of the posterior malleolar rim in males and females, respectively. (b) Distal screw placement. Screw should be inserted in at least $18^{\circ}$ and $15^{\circ}$ anterosuperior direction for males and females, respectively. about the place and the direction of the most distal screws to prevent tibiotalar joint penetration. ${ }^{[12]}$

This study aimed to determine the anatomic safe place for the screws during the fixation of PM fractures. Results of the study showed that if a screw placement parallel to the joint surface is desired, the screw insertion should be located $6 \mathrm{~mm}$ and $5 \mathrm{~mm}$ superior to the distal edge of the PM rim in males and females, respectively. And when more distal screw is required, the screw should be inserted in at least $18^{\circ}$ and $15^{\circ}$ AS direction for males and females, respectively (Figure 3 ).

The presented data and this practical information are useful and essential for the fixation of PM fractures. Although fixation is usually performed under fluoroscopic monitoring, the number of shots taken during surgery can be reduced, and the duration of operation can be shortened. In the operating theaters without fluoroscopy, screw penetration of the joint, which is a significant complication, can be prevented. Furthermore, the data presented here are important for the implant designs for this anatomic region, particularly PM plates.

There are some strengths and limitations of this study. The strengths are: (i) both male and female sexes were studied separately to understand the differences between sexes; (ii) all measurements were repeated twice by the same person, and the mean of two measurements was used to minimize the errors; and (iii) angle measurements were calculated mathematically by a computer program. The most important limitation of the study is that the study was conducted on the Turkish population. Therefore, the data may not reflect other communities due to possible ethnic variance.

In conclusion, to our knowledge, this is the first study that examined the articular depth of concave distal tibial joint surface and the posterior inclination angle of the PM tubercle. Parallel oriented screws 
should be inserted at least $6 \mathrm{~mm}$ and $5 \mathrm{~mm}$ above the $\mathrm{PM}$ rim in males and females, respectively. If a more distal screw is required, the screw should be inserted in at least $18^{\circ}$ and $15^{\circ}$ AS direction for males and females, respectively.

\section{Declaration of conflicting interests}

The authors declared no conflicts of interest with respect to the authorship and/or publication of this article.

\section{Funding}

The authors received no financial support for the research and/or authorship of this article.

\section{REFERENCES}

1. Juto H, Nilsson H, Morberg P. Epidemiology of Adult Ankle Fractures: 1756 cases identified in Norrbotten County during 2009-2013 and classified according to AO/OTA. BMC Musculoskelet Disord 2018;19:441.

2. Elsoe R, Ostgaard SE, Larsen P. Population-based epidemiology of 9767 ankle fractures. Foot Ankle Surg 2018;24:34-9.

3. Jaskulka RA, Ittner G, Schedl R. Fractures of the posterior tibial margin: their role in the prognosis of malleolar fractures. J Trauma 1989;29:1565-70.

4. Kochai A, Türker M, Çiçekli Ö, Özdemir U, Bayam L, Erkorkmaz Ü, et al. A comparative study of three commonly used fixation techniques for isolated medial malleolus fracture. Eklem Hastalik Cerrahisi 2018;29:104-9.

5. Bartoníček J, Rammelt S, Tuček M, Naňka O. Posterior malleolar fractures of the ankle. Eur J Trauma Emerg Surg 2015;41:587-600.

6. Ogilvie-Harris DJ, Reed SC, Hedman TP. Disruption of the ankle syndesmosis: biomechanical study of the ligamentous restraints. Arthroscopy 1994;10:558-60.

7. Gardner MJ, Brodsky A, Briggs SM, Nielson JH, Lorich DG. Fixation of posterior malleolar fractures provides greater syndesmotic stability. Clin Orthop Relat Res 2006;447:165-71.

8. Miller AN, Carroll EA, Parker RJ, Helfet DL, Lorich DG. Posterior malleolar stabilization of syndesmotic injuries is equivalent to screw fixation. Clin Orthop Relat Res 2010;468:1129-35.

9. Odak S, Ahluwalia R, Unnikrishnan P, Hennessy M, Platt S. Management of posterior malleolar fractures: A systematic review. J Foot Ankle Surg 2016;55:140-5.

10. Anwar A, Lv D, Zhao Z, Zhang Z, Lu M, Nazir MU, et al. Finite element analysis of the three different posterior malleolus fixation strategies in relation to different fracture sizes. Injury 2017;48:825-32.

11. Anwar A, Zhang Z, Lv D, Lv G, Zhao Z, Wang Y, et al. Biomechanical efficacy of AP, PA lag screws and posterior plating for fixation of posterior malleolar fractures: a three dimensional finite element study. BMC Musculoskelet Disord 2018;19:73.

12. Hoogendoorn JM. Posterior Malleolar Open Reduction and Internal Fixation Through a Posterolateral Approach for Trimalleolar Fractures. JBJS Essent Surg Tech 2017;7:e31. 